\title{
11 Medizinethische Entscheidungen in der geburtshilflichen Notfallmedizin
}

Martin Langer

\subsection{Einleitung ${ }^{10}$}

In der Geburtshilfe im Allgemeinen trifft man ethische Probleme sehr häufig an; Beispiele dafür sind der Schwangerschaftsabbruch aus embryopathischer Indikation, die Reduktion von höhergradigen Mehrlingsschwangerschaften oder Entscheidungen an der Grenze der Lebensfähigkeit bei extremer Frühgeburtlichkeit. Im Rahmen der geburtshilflichen Notfallmedizin jedoch scheinen rein medizinische Fragestellungen alle anderen Überlegungen bei weitem zu überwiegen. Quantitativ ist dieser Eindruck sicher richtig, in den seltenen Fällen, in denen es allerdings zu einem tatsächlichen ethischen Konflikt kommt, ist dieser meist besonders schwerwiegend und von höchster Brisanz. Es ist daher durchaus gerechtfertigt, im Folgenden einige Gedanken dazu zu formulieren.

\subsection{Charakteristik medizinethischer Probleme}

Ethische Probleme entstehen im Wesentlichen aus einem Konflikt mit einem der zwei Grundprinzipien Patientenautonomie und Benefizienz, also dem Nutzen oder Vorteil für den Patienten. Vereinfacht gesprochen, besteht ein Autonomiekonflikt dann, wenn die Patientin einer vom Arzt (dringend) vorgeschlagenen Therapie nicht zustimmt, in selteneren Fällen auch dann, wenn sie eine Therapie fordert, für die es

10 Bei geschlechtssensitiven Bezeichnungen sind immer beide Geschlechter gemeint. 
aus ärztlicher Sicht keine vernünftige Rationale gibt. Beim Benefizienzkonflikt sind sich sowohl Patientin und Arzt im Unklaren darüber, ob eine mögliche Therapie eine sinnvolle Wahrscheinlichkeit von Heilungsaussichten verspricht; der Begriff „Dilemma “ wird hier zu Recht verwendet. Stellt sich die Problematik in einer milderen, nicht-kontroversiellen Form, so bieten sich mit „shared decision making“- und ausführlichem ,informed consent“-Prozess zwischen Patientin und Arzt hilfreiche Modelle (s. Kap. 11.7). Typische Konstellationen, die so gelöst werden können, sind Entscheidungen zum Schwangerschaftsabbruch aus embryopathischer Indikation oder zum Geburtsmodus.

Weitere Prinzipien der Medizinethik, mit denen es Konflikte geben kann, ist die relative Allokation (Zuteilung) finanzieller Mittel zwischen verschiedenen Patientengruppen; der paradigmatische Konflikt diesbezüglich sind aufwendige herzchirurgische Operationen bei Neugeborenen mit Trisomie 18. Manchmal mischen sich Autonomie- und Benefizienzprinzip, etwa wenn die Bewertung der Verhältnismäßigkeit zwischen Nutzen und Risiko/Aufwand einer Therapie zwischen Arzt und Patientin stark divergiert; typische Konstellationen hierfür sind Eizellspende bei Frauen > 50 Jahre oder Bluttransfusionen bei Zeugen Jehovas, letzteres zwar juristisch klar geregelt, medizinethisch aber problematisch.

\subsection{Notfallmedizin}

Im Vergleich zu anderen geburtshilflichen Betreuungskonstellationen zeichnet sich die Notfallmedizin durch mehrere Besonderheiten aus.

Praktisch immer besteht Zeitdruck, und zwar einerseits durch die Natur der medizinischen Probleme und andererseits durch den Arbeitsdruck bei hohen Patientinnenzahlen und raschem „Durchschleuszeiten“. Die Arbeit findet darüber hinaus oft in räumlich, personell und apparativ beschränkten Verhältnissen statt.

Die Patientinnen präsentieren sich häufig mit unspezifischen Beschwerden, denen in der überwiegenden Mehrzahl keine schwerwiegenden Diagnosen zugrunde liegen. In einigen wenigen Fällen verbergen sich aber doch abklärungswürdige Erkrankungen, die es gilt, mit limitierten Mitteln und in limitierter Zeit treffsicher zu identifizieren. Neben dieser „Aufmerksamkeits-Problematik“ bei Bagatellfällen gibt es die seltenen, aber spektakulären Notfälle, die manchmal zu einer „ÜberforderungsProblematik" bei Katastrophen führen können.

Beiden ist gemeinsam und für Notfallambulanzen typisch, dass es zu keiner ausreichenden Arzt-Patientinnen-Beziehung kommen kann, innerhalb derer eine für die Entscheidungsfindung notwendige Kommunikation stattfindet. Sehr oft bestehen sprach- und kulturelle Probleme zwischen Personal und Patientinnen und/oder Schock bei den Patientinnen. Die primäre Aufgabe des Arztes wird oft sein, rasch die Natur des Hauptproblems zu identifizieren: Liegt ein im engeren Sinn des Wortes medizinischer Notfall vor oder steht vielmehr ein ethisches, forensisches, psychosomatisch/psychiatrisches oder kulturelles Problem im Vordergrund? Wenn sich, wie so oft, mehrere Aspekte überschneiden, was sind die relativen Anteile und die jeweilige Dringlichkeit?

Gelten alle diese Überlegungen bereits in Ländern mit einem hoch entwickelten Gesundheitssystem, so sind die Verhältnisse in Schwellenländern oder armutsgefähr- 
deten Regionen oft unzumutbar. Der Bedarf an „emergency obstetrics“ wird dort in keiner Weise gedeckt, und es existieren enorme Unterschiede zwischen Ländern mit unterschiedlichem Einkommensniveau (Holmer et al. 2015).

\subsection{Benefizienzkonflikte}

\subsubsection{Perimortem caesarean delivery (PMCD)}

Das paradigmatische medizinethische Dilemma in der Notfallmedizin ist jenes der Sectio bei mütterlichem Herz/Kreislaufstillstand. In älteren Lehrbüchern wurde dies als „Sectio in mortua“ bezeichnet, heute wird der korrektere Ausdruck „perimortal“ verwendet, weil es sich praktisch immer um ein Reanimationsszenario handelt. Die medizinethische Frage ist eine klassische Benefizienzproblematik, bei der folgende Güter gegeneinander abgewogen werden müssen:

- Wie hoch ist die Chance des Kindes auf ein mehr oder minder schadensfreies Überleben vs. der Gefahr einer schweren hypoxischen Schädigung bzw. dem intrauterinen Fruchttod (IUFT)? Die Bewertung eines kindlichen Überlebens mit Dauerschäden wird weiters verschärft durch das Wissen um den (wahrscheinlichen) Tod der Mutter.

- Besteht ein zusätzliches Eingriffsrisiko für die Mutter in einer Reanimationssituation vs. kann die Entleerung des Uterus die schwer beeinträchtigte Zirkulation der Schwangeren eventuell sogar entlasten?

Als grundsätzliche Orientierung wird häufig die 4 (5) Minuten-Regel angegeben, die besagt, dass 4 Minuten nach erfolgloser Reanimation mit der Notfallsectio begonnen werden müsse, um das Kind nach 5 Minuten zu entbinden, weil es bereits nach 5 Minuten zu irreversibler Hypoxie sensibler Hirnareale kommen kann.

Empirische Überprüfung dieser „Eminence-basierten“ Meinung förderte folgende Kritikpunkte zutage: Unter realistischen Bedingungen liegen die Zeiten von Kreislaufstillstand bis Geburt viel länger, nämlich bei 1o min für diejenigen, die schadensfrei überlebten, und bei $25 \mathrm{~min}$ bei den später verstorbenen Patientinnen. Ähnlich verhielt es sich bei den Neugeborenen: Schadensfreies Überleben fand sich nach 10 min, Versterben bzw. massive Schäden nach 20 min (Benson et al. 2014).

Daraus lässt sich schließen, dass es keine nachweisbare Schwelle für das Eintreten einer Hirnschädigung und somit für oder gegen eine Notfallsectio bei 4-5 min gibt, sondern die Chancen für Mutter und Kind nach dem Herz/Kreislaufstillstand kontinuierlich mit der Zeit abnehmen.

Diese Fakten stellen eine gute Orientierung für die medizinethische Bewertung derartiger Krisensituationen dar (Drukker et al. 2014; Lipman et al. 2014); typische Beispiele hierfür sind Frauen nach Herzinfarkt oder Pulmonalembolie. Nachdem selbst bei komplettem Kreislaufstillstand der Schwangeren das Kind noch 10-15' basal sauerstoffversorgt ist, ist bis dahin rasches und beherztes Vorgehen im Sinne einer schnellstmöglichen Notfallsektio per medianer Unterbauchlaparatomie die Methode der Wahl. Dabei wird die Hautschnitt-Entwicklungszeit unter 1 Minute liegen können. 
Jenseits einer Kreislaufstillstandsdauer von > 15' müssen sich die Verantwortlichen rasch folgende Information einholen:

- Zeitpunkt des mütterlichen Ereignisses bzw. der Asystolie, Beginn einer ausreichenden und lückenlosen Reanimation

- Informationen über das Gestationsalter, wobei unterhalb der SSW 28 die Frühgeburtlichkeit massiv erschwerend zur Hypoxie tritt

- Orientierender abdomineller Ultraschall hinsichtlich einer positiven Herzaktion des Kindes > 6o bpm

v Basisinformation über eine evt. Minderperfusion vor dem terminalen Ereignis

Nach der Kindesentwicklung wird der Neonatologe eine ähnliche medizinethische Bewertung des Neugeborenen durchführen müssen; die Parameter dabei sind (vermutetes) Gestationsalter, allgemeine Reife, (vermutete) Zeit der Hypoxie, APGARScore, Laborparameter wie Nabelarterien-pH, Laktat, base-excess, $\mathrm{O}_{2}, \mathrm{CO}_{2}$. Vor allem aber wird der erfahrene Pädiater im Sinne der „provisional care“ das Ansprechen des Kindes auf eine initiale, 5-10-minütige Reanimation beurteilen und als Ergebnis davon die Reanimation in eine neonatale Intensivtherapie überführen oder aber zu „comfort care“ wechseln.

Bei unbekannter Dauer, unzureichender Reanimation, Gestationsalter $<$ SSW 28 und kindlicher Herzaktion < 60 bpm ist die Wahrscheinlichkeit eines schadensfreien Überlebens des Kindes sehr gering und es kann im interdisziplinären Konsil entschieden werden, auf die perimortale Sectio aus kindlicher Indikation zu verzichten.

In einem nächsten Bewertungsschritt muss die mütterliche Indikation erwogen werden. Schwangere haben global bessere Überlebenschancen bei Reanimation als Nicht-Schwangere, und zwar 36,9\% bzw. 25,9\% (Lavecchia u. Abenhaim 2015; Tirkkonenet al. 2015). Bei einer Rate von mehr als $1 / 3$ Überleben ist daher bei diesen im wesentlich jungen und häufig vorher gesunden Frauen auf jeden Fall eine maximale Reanimation mit allem Nachdruck zu fordern! Darüber hinaus trägt die perimortale Sectio positiv zum mütterlichen Überleben bei, nachdem sich die kardiopulmonale Funktion durch die deutliche Verkleinerung des Uterus und geringeren Bedarf an Herzminutenvolumen oft verbessert (Katz 2012; Farinelli u. Hameed 2012).

\section{Der Verzicht auf oder die Beendigung einer Reanimation bei einer Schwange- ren sollte daher nur bei Vorliegen eindeutiger neurologischer Kriterien eines Hirntodes und dann nur als Teamentscheidung fallen.}

\subsubsection{Andere Benefizienzkonflikte}

Ähnliche Überlegungen wie für den Herzkreislaufstillstand gelten für Schwangere nach Polytrauma, Verdacht auf Hirnblutung oder Insult sowie ähnliche lebensbedrohliche Zustände. Hier ist die Entscheidung gemeinsam mit der jeweils zuständigen Disziplin, wie z.B. Neurologie oder Traumatologie, zu treffen. Bei rein geburtshilflichen Notfällen, wie massiver Blutung bei Plazenta prävia, bei vorzeitiger Plazentalösung oder eklamptischem Krampfanfall ist die Notfallsectio nach Blitzintubation die Methode der Wahl. 
Die Indikation zur Notfallhysterektomie ist naturgemäß sehr zurückhaltend und nur dann zu stellen, wenn die Leitlinie für postpartale Blutung schrittweise abgearbeitet wurde und danach immer noch eine vitale Gefährdung der Patientin besteht. Zusätzliche Parameter, die in die Entscheidung miteinfließen, sind das mütterliche Alter, Zahl der Kinder und zusätzliche Erkrankungen.

\subsection{Autonomiekonflikte}

\subsubsection{Verweigerung einer vorgeschlagenen Krankenhausaufnahme/Therapie}

Kommt der Arzt zu einer begründeten und dringenden Empfehlung einer stationären Aufnahme und/oder Behandlung, die Patientin verweigert aber, so muss der Arzt neuerlich und in betont ausführlicher Form einen „informed consent-Prozess“ mit der Patientin durchlaufen. Dabei muss deutlich auf ihre wohlverstandenen Interessen im Sinne ihrer eigenen Gesundheit und der Gesundheit ihres Kindes hingewiesen werden. Beharrt sie danach weiter auf ihrem Standpunkt, muss man sich die Frage stellen, ob in der gegebenen geburtshilflichen Situation die Einsichts- und Urteilsfähigkeit der Patientin gegeben ist, wenn sie die Selbst- oder Fremdgefährdung (des Kindes) nicht erkennen kann. Bei starker Blutung, hochpathologischem CTC oder Geburt im Gange, jeweils mit lebensfähigem Kind, wird Fremdgefährdung vorliegen, bei mäßiger intrauteriner Wachstumsretardierung oder leichten vorzeitigen Wehen wohl nicht. Sollte im ersteren Fall mit der Patientin (und ihren Angehörigen) keine vernünftige Lösung ausverhandelt werden können (z.B. engmaschige Kontrolle am nächsten Tag), muss zum Schutz der Patientin und auch des Arztes selbst eine formale psychiatrische Exploration mit der ausdrücklichen Frage nach Einweisung in der Psychiatrie wegen Selbst- und/oder Fremdgefährdung eingeleitet werden.

In ganz seltenen Fällen eskaliert die Lage bzw. entweicht die Patientin. In aller Regel wird man selbst dann auf die Einleitung einer aktiven polizeilichen Suche verzichten, nur bei begründetem Verdacht auf Gewaltanwendung sollte zu dieser Ultima ratio gegriffen werden. In allen anderen Situationen muss die Selbstbestimmung und Autonomie der Patientin in unserem Rechtssystem als das höhere Rechtsgut angesehen werden. Allerdings muss dann der gesamte Ablauf exakt und eventuell mit Zeugen dokumentiert werden, um späteren Klagen begegnen zu können.

\subsubsection{Verweigerung eines dringend indizierten Kaiserschnittes („,court ordered caesarean section“ vs. „emergency coerced caesarean section“)}

Zu einer akuten Zuspitzung der oben beschriebenen Situation kann es in ganz seltenen Fällen dann kommen, wenn die Patientin eine dringlich indizierte Sectio ablehnt. Chervenak hat dafür den Begriff „erzwungene Notfallsektio“ (coerced emergency caesarean) geprägt und trifft die Unterscheidung zwischen dieser und der „Sectio auf Gerichtsbeschluss“ (court ordered caesarean) nach folgenden drei Kriterien:

1. hohe Verlässlichkeit der prognostischen Bewertung und Abwägung insofern, als die Sectio eine ernsthafte kindliche Morbidität oder Mortalität verhindern wird

2. kein physischer Widerstand, der das Risiko mütterlicher oder kindlichen Schaden durch die erzwungene Sectio erhöhen würde

3. ungenügend verfügbare Zeit, um ein Gerichtsurteil zu erwirken 
Eine derartige Extremsituation werden die meisten Gynäkologen wahrscheinlich nie in ihrer professionellen Karriere erleben und jeder Beratungs- und Überredungsaufwand ist gerechtfertigt, um sie zu verhindern. Trotzdem ist es aber sinnvoll, sie sich theoretisch durchzudenken, um andere, mildere Konstellationen daran zu messen und sie zu managen.

\subsubsection{Konflikte zwischen den Werthaltungen der Patientin und des Arztes}

Grundsätzlich ist ein Arzt berechtigt, die Medizin innerhalb eines selbst gesetzten Rahmens von medizinisch-wissenschaftlichen und ethischen Kriterien auszuüben. Jeder klinisch Tätige ist aufgerufen, seinen persönlichen Rahmen antizipierend und ohne konkreten Zeitdruck zu reflektieren, um darüber im Notfall verfügen zu können. Ebenso ist es unbestritten, dass im Konfliktfall die ethischen Werte der Patientin der zentrale und entscheidungsleitende Bezugsrahmen sind. Zwischen den ärztlichen und den Patientinnenwerten kann es zu Konflikten kommen, und zwar sowohl hinsichtlich des medizinischen als auch des ethischen Anteils des ärztlichen, subjektiven Bezugsrahmens. Je nachdem verlangen sie auch etwas unterschiedliche Lösungsmodelle.

Ein Beispiel für die diskrepante medizinische Einschätzung stellt die abgebrochene Hausgeburt dar, bei der die Gebärende dann unter Notfallbedingungen und in möglicherweise schlechtem fetalen und/oder mütterlichen Zustand in ein Krankenhaus eingeliefert wird. Nun stehen viele Geburtshelfer, und das mit guten Gründen, einer Hausgeburt sehr skeptisch gegenüber. So hat etwa Chervenak bei hebammengeleiteten Hausgeburten im Vergleich zu hebammengeleiteten Klinikgeburten ein relatives Risiko von 3,8 und in einzelnen Risikogruppen wie z.B. Erstgebärende oder Schwangere $>$ SSW 41 ein bis zu 6-fach erhöhtes RR gefunden. Weiters zeigte sich, dass bei Hausgeburten nicht nur Schwangere mit niedrigem Risiko, sondern auch solche mit Beckenendlagen, Mehrlingen oder Zustand nach Sectio entbunden wurden.

\footnotetext{
Ungeachtet dieser empirischen Daten muss der Notfallgynäkologe jedoch streng darauf achten, seine allgemeine Haltung zur Hausgeburt auf keinen Fall in die Betreuung eines konkreten Falles einfließen zu lassen. Im Gegenteil: Gefordert in dieser Situation sind ausschließlich rasche und umsichtige medizinische Hilfe und verständnisvolle Betreuung, und zwar völlig ohne Schuldzuweisungen an die Gebärende oder die begleitende Hebamme.
}

Zum korrekten Selbstschutz sind die geburtshilflichen Parameter bei der Aufnahme zu dokumentieren und der Fall in einer Besprechung mit der Hebamme aufzuarbeiten. Moralische Werthaltungen von Patientin und Arzt können in Fragen des Schwangerschaftsabbruches und der Kontrazeption kollidieren. In manchen katholisch-konfessionellen Spitälern oder Ländern (z.B. Irland, Polen) ist ein Schwangerschaftsabbruch unter praktisch allen Umständen verboten, selbst dann, wenn die Mutter gefährdet oder die Schwangerschaft/das Kind keine Überlebensperspektive hat (Freedman et al. 2008). Die Definition der schützenswerten Schwangerschaft wird dabei sehr weit gefasst, nämlich lediglich im Sinne einer positiven Herzaktion. 
Europaweit bekannt wurde der Fall einer indischen Patientin in einem irischen Spital, der bei manifestem Amnioninfektionsydrom in der SSW 18 die Entleerung des Uterus wegen noch bestehender Herzaktionen versagt wurde und die daran verstarb.

Es kann nicht klar genug betont werden, dass bei einer (vital) gefährdeten Patientin die moralische Haltung des Arztes völlig zurückzustehen hat und ausschließlich diejenige der Patientin und die medizinische Situation zu berücksichtigen sind. Sollte die Patientin noch transportfähig sein, wäre der rasche Transfer an eine Abteilung, die den notwendigen Eingriff durchführen würde, eine gerade noch gangbare Kompromisslösung. In diesem Fall hat aber der verweigernde Notfallarzt persönlich den Kontakt mit der anderen Abteilung aufzunehmen und verlässlich den Rettungstransport zu organisieren. Seine Verantwortung endet erst dann, wenn die Patientin nachweislich in der anderen Abteilung aufgenommen wurde.

Weniger dramatisch gestaltet sich der Wunsch nach post-koitaler Kontrazeption, wobei im Jahr 2015 die Akzeptanz der Methode in öffentlichen Spitälern gegeben sein sollte, nachdem die gesetzliche Lage eine Abgabe in Apotheken und Spitälern erlaubt (Greene 2008; Mittal 2014). In der Großstadt hätte die Patientin meist mehrere Alternativen, in ländlichen Gegenden kann dies hingegen schon schwieriger sein. Allgemein sollte sich ein Gynäkologe, der eine sehr fundamentalistische, ablehnende Einstellung zur Kontrazeption einnimmt, fragen, ob dies mit seiner Berufsausübung in der heutigen Zeit kompatibel ist.

\subsection{Andere Konfliktfelder}

Notfallambulanzen sind natürlich auch Anlaufstellen für Opfer von jeglicher Form von Gewalt: Häusliche, (sexualisierte) Gewalt oder Kindesmisshandlung (Hoelle et al. 2015) sind die wichtigsten Beispiele hierfür. Der Erstaufnahmearzt sieht sich vor dem Dilemma, entweder Cewaltfolgen zu übersehen und damit die Chance, den Kreislauf der Gewalt zu unterbrechen oder wegen einer banalen Verletzung einen Elternteil als Täter zu desavouieren. Entscheidend sind die Kenntnis der typischen Charakteristika einer Misshandlung (wie z.B. ähnliche Verletzungen unterschiedlichen Alters, parallele Anordnung) sowie ein Gespräch mit dem Kind/der Verletzten in geschützter Atmosphäre, sei es durch eine Ärztin oder zumindest in Beisein einer weiblichen Person, eine exakte (Foto)Dokumentation, Asservierung eventueller Beweisstücke und Hinweis auf Opferschutzgruppen etc.

Bei Schwangeren, die vermutlich oder sicher zusätzlich an einer psychiatrischen Erkrankung wie Suizidalität, psychotische Krise, affektiven Störungen, Alkohol- oder Drogeneinfluss leiden, muss ohne falsche Scheu vor Stigmatisierung der Fachpsychiater beigezogen werden. Dieser muss dezidiert zu Selbst- und Fremdgefährdung Stellung nehmen, die in diesem Fall das Kind ausdrücklich miteinschließt.

In den letzten Jahren haben kulturell verursachte Konflikte in Notfallaufnahmen stark zugenommen (Van McCrary et al. 2014). Sie stellen sich entweder in der Form dar, dass die Patientin sich nicht von einem Mann untersuchen lässt, wobei diese Forderung oft von dem begleitenden Mann vorgebracht wird. In der milderen Ausprägung übernimmt der Mann paternalistisch die Rolle des Beschützers oder Sprechers der Frau, sodass nicht klar wird, was nun der Wille der Patientin ist und eine 
informierte Zustimmung in der rechtlich geforderten Form nicht einzuholen ist. Erschwert wird eine derartige Situation meist noch durch Sprachprobleme, wobei der Mann wenig und die Frau oft überhaupt nicht Deutsch oder Englisch spricht.

Zur Frage der Untersuchung durch eine Gynäkologin bestehen zwei Haltungen: In der strengen Form wird darauf verwiesen, dass es im öffentlichen Krankenhaus keine freie Arztwahl gäbe und jeder Kollege/in sich gleichermaßen bemüht, auf die Intimsphäre der Patientin Rücksicht zu nehmen. Die andere Position weist darauf hin, dass ein zumutbarer Aufwand betrieben werden kann, innerhalb eines Teams, in dem mit hoher Wahrscheinlichkeit auch Kolleginnen vertreten sind, eine davon für die Untersuchung zu verpflichten. Es wird von der konkreten Interaktion zwischen Patientin und Team, der medizinischen Diagnose, dem Alter der Patientin und organisatorischen Faktoren abhängen, welche der beiden Haltungen nun überwiegt.

Nimmt der Notfallarzt eine an der individuellen Patientin orientierte und korrektprofessionelle Haltung ein und führt die Untersuchung im Beisein einer weiblichen Zeugin durch, wird man sich leicht gegen etwaige Rassismusvorwürfe zur Wehr setzen können, die durchaus auch an der Tagesordnung in Ambulanzen stehen.

\subsection{Lösungsansätze für ethische Probleme in der geburtshilflichen Notfallmedizin}

Es muss vorausgeschickt werden, dass es für die oben besprochenen Situationen keine allgemein gültigen Lösungen gibt und geben kann. Zu vielfältig sind die Gemengelagen und denkbaren Konstellationen, zu unterschiedlich die jeweiligen Arbeitssituationen.

Weiters lässt sich feststellen, dass manche der ansonsten in Spitälern üblichen und erfolgreichen Strategien der Problemlösung in der Notfallmedizin nicht oder nur eingeschränkt zur Verfügung stehen; dazu zählen „Beiziehen eines bislang unbeteiligten Dritten“, zeitlich definierte Moratorien oder „Denkpausen“. Bei der Personalknappheit und Hektik wird es schwer sein, rasch einen von beiden Seiten akzeptierten Dritten zu finden, der mit seiner fachlichen und menschlichen Autorität entscheidend zur Lösung beitragen kann. Ähnliches gilt für Denkpausen oder Moratorien, die sich wegen der Dringlichkeit der medizinischen Probleme verbieten.

Andere aus der allgemeinen Medizinethik bekannten Strategien können hingegen auch in der Notfallsituation konstruktiv angewendet werden.

Aus der Diskursethik stammt das induktive Schlussfolgern („bottom-up“), also der Vorrang des konkreten Einzelfalles vor der Durchsetzung einer abstrakten Norm. In diesem Sinn sollte das Personal weitgehende Flexibilität und Kreativität beim Finden von eventuell auch unkonventionellen Lösungen aufbringen.

Übertriebene Sorge vor der Verletzung von Dienstvorschriften ist nicht angebracht, mithilfe exakter Dokumentation einschließlich der Schilderung der Ausgangssituation und der handlungsleitenden Überlegungen kann unangenehmen Nachforschungen souverän begegnet werden. 
Typische Beispiele für diese Vorgangsweise sind bei Verweigerung einer Therapie, wo vielleicht ambulante Kontrollen anstelle der ursprünglich vorgesehenen stationären Aufnahme vereinbart werden können, wenn die Patientin grundsätzlich paktfähig wirkt.

Ob und in welcher Form die Angehörigen einer Patientin in die Betreuung eingebunden werden sollen, hängt stark von der medizinischen Dringlichkeit, dem Bewusstseinszustand und den Sprachkenntnissen der Patientin ab. Grundsätzlich sollten Kinder nicht als Dolmetscher herangezogen werden, sondern professionelle Dolmetscher dies übernehmen. Im Akutfall und in der Nacht wird man allerdings jede denkbare Verständigungsmöglichkeit nützen. Es sollte ganz klar zwischen jenen Entscheidungen unterschieden werden, die in die ärztliche Verantwortung fallen und anderen, bei denen die Angehörigen ein gewisses Mitspracherecht besitzen. Weiters gibt es Situationen, wo eben das Gegenteil, nämlich das Ausschließen der Angehörigen und das ungestörte Gespräch mit der Patientin allein unabdingbar sind; so etwa beim Verdacht auf häusliche Gewalt oder Kindesmissbrauch.

Bei sehr besorgten oder erregten Angehörigen droht eine Interaktion manchmal außer Kontrolle zu geraten. So schwierig es auch sein mag, muss der Arzt versuchen zu de-eskalieren, etwa durch klare, feste Sprache, mit der aber Verständnis für die nervliche Anspannung signalisiert wird, durch Hereinholen einer Krankenschwester etc. Es muss ganz klar sein, dass die Patientin sich in Spitalspflege begeben hat und das Personal seine Pflichten wahrnehmen wird, dass aber auch die Patientin die Pflicht zur Kooperation hat.

Die Patientenautonomie stellt zwar ein zentrales Prinzip der Medizinethik dar, es ist aber wichtig zu betonen, dass sie nicht von vornherein als gegeben vorliegt, sondern erst durch den „informed consent-Prozess“ hergestellt werden muss (Langer 2011). Der Umfang der Patientenaufklärung hängt wesentlich von der Tragweite und Dringlichkeit des Eingriffs ab; je dringlicher und vor allem unter Notfallbedingungen darf der informed consent-Prozess kursorisch sein (Moore et al. 2014). Im Falle von Komplikationen und einer Rechtsklage müsste die Patientin nämlich den Beweis erbringen, dass sie der Handlung nicht zugestimmt hätte, wenn sie ausführlicher über eventuelle Nebenwirkungen aufgeklärt worden wäre.

Bei bewusstloser und/oder vital gefährdeter Patientin oder im Extremfall bei permanent vegetativen Status kommt das Konzept des „substituted judgement“ (mutmaßlicher Wille) der Patientin zur Anwendung. Dieses nimmt als Ausgangspunkt an, dass die Absicht und der Wunsch der gesunden Schwangeren vor dem Ereignis jener nach einem möglichst gesunden Kind war und dies daher auch danach angenommen werden darf. Dabei kann es durchaus gerechtfertigt sein, die Absichten der Schwangeren anhand ihrer eigenen, zielgerichteten Handlungen zu hinterfragen und zu konkretisieren. So etwa darf bei regelmäßigen, dokumentierten Vorsorgeuntersuchungen inklusive Screening-Ultraschallkontrollen fraglos ein zukunftsgerichtetes, fürsorgliches Handeln zugunsten des Kindes unterstellt werden. Ein Präzedenzfall für diese Fragestellung war vor Jahren das sogenannte „Erlanger Baby“. Dabei verfiel eine junge Schwangere im II. Trimenon nach einem Verkehrsunfall in ein irreversibles Hirnversagen (Hirntod). Als Beweis, dass sie eine Haltung „Pro Kind“ einnähme, wurde angeführt, dass sie die Frist für einen evt. Schwangerschaftsabbruch verstreichen hatte lassen. Diese Position, die letztlich zu einer Frau im permanent-vegetativen Zustand als „Brutkasten“ geführt hätte, wurden m.E.n. zu Recht heftig kritisiert. 
Beim Fall des sogenannten „Erlanger Babys“ wurde hingegen zu Recht bezweifelt, ob allein das Verstreichenlassen der Frist zur Abtreibung bereits ein fürsorgliches Handeln darstellt.

\subsection{Präventive Ethik}

Zwei pragmatische Alltagsstrategien sollten berücksichtigt werden: Wann immer personell möglich, sollte für einen Notfalleingriff wie eine perimortem caesarean section das „best team available“, unabhängig von etwaigen Dienstzuteilungen, zusammengestellt werden. Wenn der betreuende Arzt nach reiflicher Überlegung zu dem Schluss kommt, dass er den von der Patientin gewünschten Eingriff nicht durchführen kann/will, ist er dazu verpflichtet, die Patientin an einen Kollegen, der dies evtl. durchführt, zu überweisen; das schließt sogar Adressen im Ausland mit ein.

Die am ehesten Erfolg versprechende Strategie sind Simulationstrainings des gesamten Teams, die auch ethische Fragen beinhalten sollten (Bogne et al. 2014; Smith et al. 2012). Für andere Situationen, wie Notfälle im Kreißsaal oder der neonatologischen Reanimation, konnte nämlich gezeigt werden, dass professionell durchgeführte Teamtrainings eindeutige Erfolge zeitigen (Sorensen 2007).

Zu den kennzeichnenden Features solcher Drills gehören: ein nicht zum Team gehöriger, ausgebildeter Leiter, Bereitschaft und Freiwilligkeit der Teilnehmer, geschützte Atmosphäre ohne Hierarchie, keine Schuldzuweisungen, Vor- und Nachbesprechung; gute technische Ausstattung wie realistischer Schauplatz, Videoaufnahmen, die bei der Nachbesprechung analysiert werden.

\section{Literatur}

Benson MD, Padovano A, Bourjeily G, Zhou Y (2014) Perimortem Cesarean Delivery: Injury-Free Survival as a Function of Arrest-to-Delivery Interval Time. Obstet Gynecol. 123(1): 137S

Bogne V, Kirkpatrick C, Englert Y (2014) Simulation training in the management of obstetric emergencies. A review of the literature. Rev Med Brux. 35(6): 491-8

Chervenak FA, McCullough LB, Skupski DW (1993) An ethical justification for emergency, coerced cesarean delivery. Obstet Gynecol. 82(6): 1029-35

Drukker L, Hants Y, Sharon E, Sela HY, Grisaru-Granovsky S (2014) Perimortem cesarean section for maternal and fetal salvage: concise review and protocol. Acta Obstet Gynecol Scand. 93(10): 965-72

Farinelli CK, Hameed AB (2012) Cardiopulmonary resuscitation in pregnancy. Cardiol Clin 30(3): 453-61

Freedman LR, Landy U, Steinauer I (2008) When there's a heartbeat: miscarriage management in Catholic-owned hospitals. Am J Public Health 98(10): 1774-8

Greene MF (2008) Emergency contraception: a reasonable personal choice or a destructive societal influence? Clin Pharmacol Ther 83(1): 17-9

Hoelle RM, Elie MC, Weeks E, Hardt N, Hou W, Yan H, Carden D (2015) Evaluation of healthcare use trends of highrisk female intimate partner violence victims. West J Emerg Med. 16(1): 107-13

Holmer H, Oyerinde K, Meara I, Gillies R, Liljestrand I, Hagander L (2015) The global met need for emergency obstetric care: a systematic review. BJOG 122(2): 183-9

Katz VL (2012) Perimortem cesarean delivery: its role in maternal mortality. Semin Perinatol. 36(1): 68-72

Langer M (2011) Ethische Probleme in der Geburtshilfe. In: Schneider H, Husslein P-W, Schneider KTM (Hrsg.) Die Geburtshilfe, 4. Auflage Springer, Heidelberg, S. 1168-1181

Lavecchia M, Abenhaim H (2015) Cardiopulmonary resuscitation of pregnant women in the emergency department. Resuscitation pii: S0300-9572(15)00035-0 
Lipman S, Cohen S, Einav S, Jeejeebhoy F, Mhyre JM, Morrison LJ, Katz V, Tsen LC, Daniels K, Halamek LP, Suresh MS, Arafeh I, Gauthier D, Carvalho IC, Druzin M, Carvalho B; Society for Obstetric Anesthesia and Perinatology (2014) The Society for Obstetric Anesthesia and Perinatology consensus statement on the management of cardiac arrest in pregnancy. Anesth Analg. 118(5): 1003-16

Mittal S (2014) Emergency contraception - Potential for women's health. Indian I Med Res. 140 Suppl: S45-52

Moore GP, Moffett PM, Fider C, Moore MJ (2014) What emergency physicians should know about informed consent: legal scenarios, cases, and caveats. Acad Emerg Med. 21(8): 922-7

Smith A, Edwards S, Siassakos D (2012) Effective team training to improve outcomes in maternal collapse and perimortem caesarean section. Resuscitation 83(10): 1183-4

Sorensen SS (2007) Emergency drills in obstetrics: reducing risk of perinatal death or permanent injury. JONAS Healthc Law Ethics Regul. 9(1): 9-16; quiz 17-8

Sweeney I, Maietta R, Olson K (2015) An analysis comparing "sim huddles" to traditional simulation for obstetric emergency preparedness. Nurs Womens Health 19(1): 16-25

Tirkkonen I, Olkkola KT, Tenhunen I, Hoppu S (2015) Ethically justified treatment limitations in emergency situations. Eur I Emerg Med. 2015 lan 30 [Epub ahead of print]

Van McCrary S, Green HC, Combs A, Mintzer JP, Quirk IG (2014) A delicate subject: The impact of cultural factors on neonatal and perinatal decision making. I Neonatal Perinatal Med. 7(1): 1-12

\section{Univ.Prof. Dr. med. Martin Langer}

Facharzt für Gynäkologie und Geburtshilfe. Medizinstudium in Wien und Montpellier. Habilitation 1991 mit der Monographie „Somatopsychische Gynäkologie“.

Leitender Oberarzt der Abteilung für Geburtshilfe und Fetomaternale Medizin, Medizinische Universität Wien, Österreich. Systemischer Psychotherapeut. Ehem. Mitglied der Ethikkommission der MedUni Wien. 